\title{
Specific Antibodies for Coeliac Disease in Patients with Crohn's Disease in Two University Centers in Poland
}

ISSN: 2637-7632

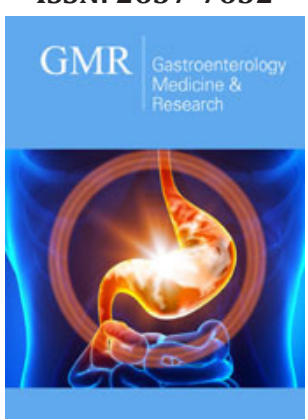

*Corresponding author: Anna Szaflarska-Popławska, Department of Pediatric Endoscopy and Gastrointestinal Function Testing, Collegium Medicum in Bydgoszcz, Poland

Submission: 非 August 09, 2019

Published: 佣August 20, 2019

Volume 3 - Issue 4

How to cite this article: Szaflarska $P$ A, Kłopocka M, Liebert A, Konopka E, Trojanowska I, et al. Specific Antibodies for Coeliac Disease in Patients with Crohn's Disease in Two University Centers in Poland. Gastro Med Res. 3(4). GMR.000566. 2019.

DOI: $10.31031 /$ GMR.2019.03.000566

Copyright@ Anna Szaflarska-Popławska, This article is distributed under the terms of the Creative Commons Attribution 4.0 International License, which permits unrestricted use and redistribution provided that the original author and source are credited.

\author{
Szaflarska Popławska $A^{1 *}$, Kłopocka $\mathbf{M}^{2}$, Liebert $A^{2}$, Konopka $E^{3}$, Trojanowska \\ $\mathrm{I}^{3}$, Bierła $\mathrm{J}^{3}$ and Cukrowska $\mathrm{B}^{3}$ \\ ${ }^{1}$ Department of Pediatric Endoscopy and Gastrointestinal Function Testing, Poland \\ ${ }^{2}$ Department of Gastroenterology and Nutrition Disorders, Poland \\ ${ }^{3}$ Department of Pathology, Poland
}

\begin{abstract}
The previous studies assessing prevalence of coeliac disease in patients with Crohn's disease have produced conflicting results. Moreover, anti-tissue transglutaminase antibodies which are implemented in the diagnostic scheme of coeliac disease seem to have limited value for the diagnosis in Crohn's disease patients. So, we would like to share with the readers our experience of the study in 104 Crohn's disease patients, who were tested for anti-tissue transglutaminase antibodies, anti-tTG, anti-deamidated gliadin peptides antibodies, anti-DGP and anti-endomysium antibodies, EMA. We think that coeliac disease occurs among Polish patients with Crohn's disease with comparable prevalence to the general population. Moreover, anti-tTG, anti-DGP and EMA seem to be equally valuable for coeliac disease screening in patients with Crohn's disease.
\end{abstract}

Keywords: Coeliac disease; Crohn's disease; Screening

\section{Introduction}

Coeliac disease (CeD) and Crohn's disease (CD) are both chronic immune-mediated inflammatory bowel disorders, which manifest with overlapping gastrointestinal and extraintestinal symptoms such as chronic diarrhea, abdominal pain and failure to thrive. Both conditions may be associated with similar small intestinal lesions, including villous atrophy and intraepithelial lymphocytosis. These clinical and histopathological features make it challenging to diagnose $\mathrm{CeD}$ in $\mathrm{CD}$ patients [1].

The previous studies assessing the prevalence of $\mathrm{CeD}$ in patients with $\mathrm{CD}$ have produced conflicting results. Some authors believe that despite the increased positivity for serum coeliac-specific antibodies in CeD, no true association between both $\mathrm{CeD}$ and CD exists [1$3]$. However, according to the recently published systematic review the risk for CeD among adult patients with inflammatory bowel disease is twice as high as in general population and this effect seems to be comparable for ulcerative colitis and CD. In contrast, the prevalence of inflammatory bowel disease in CeD patients has been described as eleven times higher than in general population [4]. In children, there are only a few case reports and small sample studies assessing coeliac serology in pediatric patients with CD [5,6]. As genetic factors are involved in the pathogenesis of both $\mathrm{CD}$ and $\mathrm{CeD}$, some authors have tried to identify share common risk loci. The HLA-DQ2 and HLA-DQ8 alleles contributing to the CeD risk have been recently ruled out as the high-risk alleles for inflammatory bowel diseases and some of them, namely HLA-DQ8 may even protect from the development of CD [7]. However, four non-HLA loci: PTPN2, IL18RAP, TAGAP, and PUS 10 have all demonstrated genome-wide association with both $\mathrm{CeD}$ and CD [8]. Interestingly, coexisting CeD seems not to have an effect on the natural course or phenotype of CD [9].

Currently, the serological screening for CeD is based on highly sensitive and specific antibodies belonging to IgA and IgG classes, namely anti-tissue transglutaminase antibodies (anti-tTG), anti-endomysium antibodies (EMA) and anti-deamidated gliadin peptide antibodies (anti-DGP). The most sensitive serological marker for CeD is represented by IgAtTG, therefore, it has been implemented in the diagnostic scheme of the disease. On the other hand, IgA-EMA have the highest diagnostic specificity if tested in expert laboratories [10]. 
Conflicting results concerning the diagnostic accuracy of these antibodies for the $\mathrm{CeD}$ diagnosis in patients with autoimmune disorders other than $\mathrm{CeD}$ have been reported. A high rate of falsepositive coeliac-specific anti-tTG in patients with inflammatory bowel diseases has previously been confirmed $[2,11]$. Interestingly, the diagnostic utility of food-related antibodies, namely anti-DGP, has been shown to be comparable or even higher in CeD patients when compared to the standard tissue transglutaminase testing [12].

We would like to share with the readers the results of our prospective study in 104 consecutive pediatric and adult patients with CD diagnosed according to clinical, radiological, endoscopic and histopathological criteria (39 females, 65 males, mean age 26.3 years, range 8-64 years) from two tertiary university units in Bydgoszcz, Poland. In order to evaluate the occurrence of specific antibodies for CeD in all patients we assessed anti-tTG and antiDGP measured by chemiluminescence assay (Thermo Fisher) and EMA measured by indirect immunofluorescence in both IgA and IgG classes. Anti-tTG and anti-DGP concentrations > 10 units (AU/ $\mathrm{mL}$ ) and EMA titer $>$ 1:5 were considered as positive. Among the studied patients only one adult 38-year-old woman had elevated IgA-tTG (>100 AU/ml), IgA-DGP (>100 AU/ml), IgG-DGP (98 $\mathrm{AU} / \mathrm{ml})$ and IgA-EMA (1:3200) and eventually duodenal biopsy revealed chronic duodenal inflammation corresponding to Marsh $3 \mathrm{c}$ mucosal lesions. All the other patients displayed negative results in all types of antibodies. Hence, the prevalence of serologically identified CeD was 1 in 104 which is comparable to the general population in Poland (1:124) [13]. On this basis, we concluded that the prevalence of $\mathrm{CeD}$ among Polish patients with $\mathrm{CD}$ is to low to motivate screening for this condition in the regular workup of patients with Crohn's disease. Moreover, anti-tTG, anti-DGP and EMA seem to be equally valuable for CeD screening in patients with CD.

\section{References}

1. Watanabe Ch, Komoto S, Hokari R, Kurihara Ch, Okada Y, et al. (2014) Prevalence of serum celiac antibody in patients with IBD in Japan. J Gastroenterol 49(5): 825-834.
2. Kocsis D, Tóth Z, Csontos AA, Miheller P, Pák M, et al. (2015) Prevalence of inflammatory bowel disease among coeliac disease patients in a Hungarian coeliac center. BMC Gastroenterology 15: 141.

3. Jandaghi E, Hojatnia M, Vahedi H, Shahbaz-Khani B, Kolahdoozan S, et al. (2015) Is the prevalence of celiac disease higher than the general population in inflammatory bowel disease? Middle East J Dig Dis 7(2): 82-87.

4. Shah A, Walker M, Burger D, Martin N, Wulffen M, et al. (2019) Link between celiac disease and inflammatory bowel disease. J Clin Gastroenterol 53(7): 514-522.

5. Alper A, Rojas-Velasquez D, Pashankar S (2018) Prevalence of antitissue transglutaminase antibodies and celiac disease in children with inflammatory bowel disease. JPGN 66(6): 934-936.

6. Virta LJ, Kolho KL (2013) The risk of contracting pediatric inflammatory bowel disease in children with celiac disease, epilepsy, juvenile arthritis and type 1 diabetes-a nationwide study. J Crohns Colitis 7(1): 53-57.

7. Watts MM, Minguez M, Planelles D, Navarro S, Rodrigez A, et al. (2018) HLA-DQ: celiac disease vs inflammatory bowel disease. World J Gastroenterol 24(1): 96-103.

8. Festen EAM, Goyette P, Green T, Boucher G, Beauchamp C, et al. (2011) A meta-analysis of genome-wide association scans identifies IL18RAP PTPN2, TAGAP, and PUS10 as shared risk loci for Crohn's disease and celiac disease. PLoS Genetics 7(1): e1001283.

9. Oxfort EC, Nguyen DD, Sauk J, Korzenik JR, Yajnik V, et al. (2013) Impact of co-existent celiac disease on phenotype and natural history of inflammatory bowel diseases. Am J Gastroenterol 108(7): 1123-1129.

10. Caio G, Volta U, Sapone A, Leffler DA, De Giorgio R, et al. (2019) Celiac disease: A comprehensive current review. BMC Medicine 17(1): 142.

11. Cabral VL, Patricio FR, Ambrogini O, Miszputen S (2011) Anti-tissue transglutaminase antibodies (IgA and IgG) in both Crohn's disease and autoimmune diabetes. Rev Esp Enferm Dig 103(9): 453-457.

12. Hoerter NA, Shannahan SE, Suarez J, Lewis SK, Green PHR, et al. (2017) Diagnostic yield of isolated deamidated gliadin peptide antibody elevation for celiac disease. Dig Dis Sci 62(5): 1272-1276.

13. Popławska A, Parzecka M, Müller L, Placek W (2009) Screening for celiac disease in Poland. Med Sci Monit 15(3): PH7-11. 